\title{
Perimetria computadorizada e manual em pacientes com defeitos perimétricos temporais avançados causados por tumores supra-selares
}

\author{
Computerized and manual perimetry in patientes with severe temporal visual field \\ defects due to supraselar tumors
}

\author{
Andrea Pereira ${ }^{1}$ \\ Mário Luiz Ribeiro Monteiro ${ }^{2}$
}

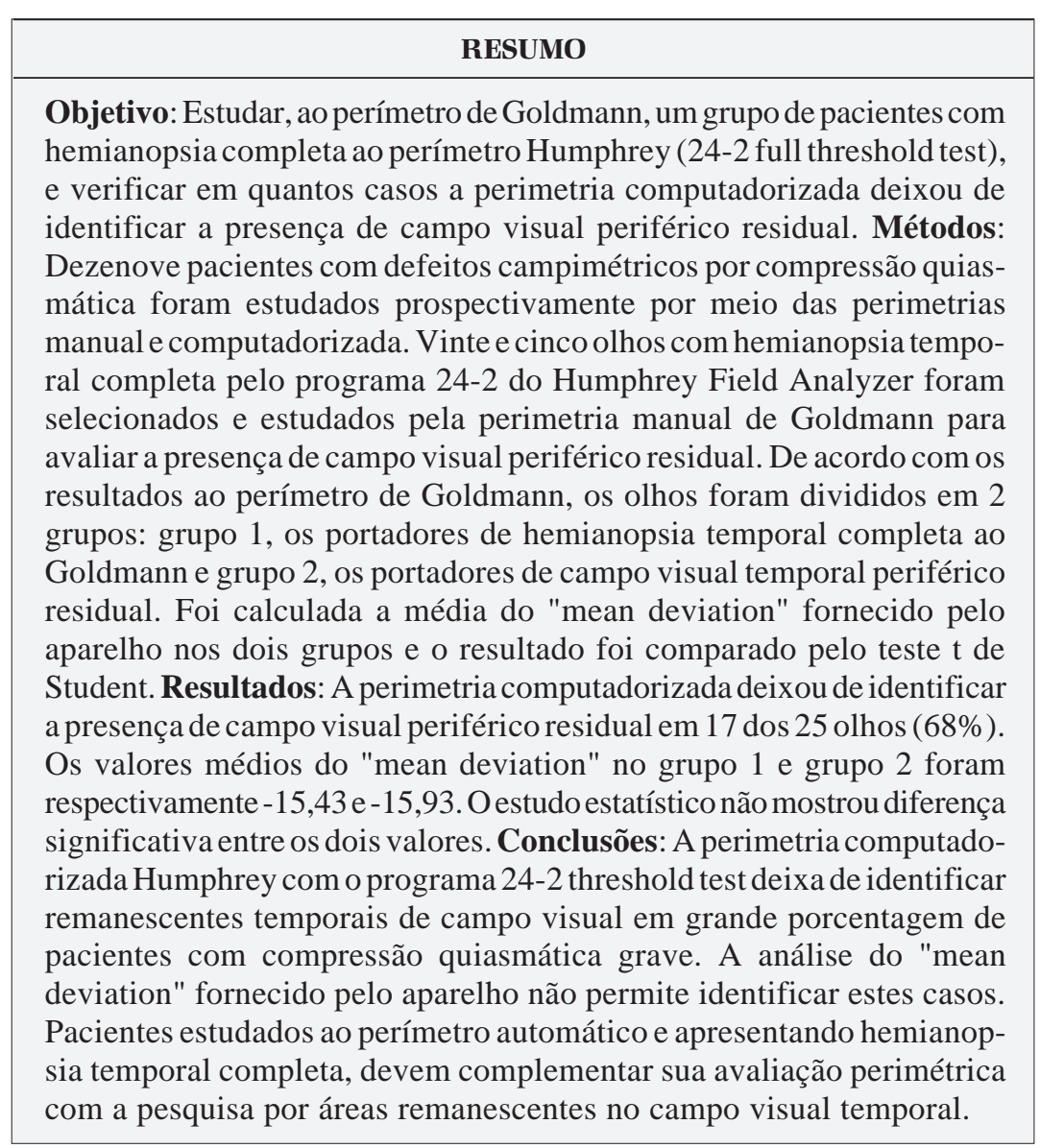

Descritores: Perimetria/métodos; Campos visuais; Quiasma óptico/patologia; Transtornos da visão/diagnóstico; Neoplasias hipofisárias; Técnicas de diagnóstico oftalmológico

\section{INTRODUÇ̃̃̃O}

O exame de campo visual é um exame propedêutico de fundamental importância na avaliação de pacientes com condições neurológicas acometendo o sistema visual. A perimetria pode ser utilizada para se detectar e quantificar anormalidades no campo visual, assim como para monitorar a resposta ao tratamento clínico ou cirúrgico apresentado pelo paciente. $\mathrm{O}$ exame pode ser feito através do emprego de vários métodos entre eles o 
teste de confrontação, o teste de tela tangente e, mais freqüentemente, através da utilização de dois tipos de perímetros: o manual e o computadorizado.

$\mathrm{Na}$ última década, a perimetria computadorizada tornou-se o exame padrão para a avaliação do campo visual ${ }^{(1)}$; a perimetria manual passou a ser um teste de certo modo obsoleto na prática diária. Apresenta vantagens em relação à perimetria manual tais como exigir menor habilidade técnica do operador, apresentar menor variabilidade quando realizada por diferentes técnicos e ser mais padronizada ${ }^{(1-2)}$. A perimetria computadorizada tem se mostrado comparável à perimetria manual na detecção da perda de campo visual no glaucoma ${ }^{(3)}$. No entanto, a sua capacidade de detectar anormalidades no campo visual e localizar a lesão nos casos de doenças neurológicas ainda não está bem determinada apesar de ser extensivamente utilizada para tal fim.

O objetivo deste trabalho é estudar um grupo de pacientes que apresentam defeito perimétrico avançado decorrente de tumores supra-selares e comparar as alterações observadas no perímetro de Goldmann com aquelas encontradas na perimetria computarizada Humphrey, com o intuito de identificar com que freqüência a perimetria computadorizada deixa de identificar a presença de campo visual periférico residual.

\section{MÉTODOS}

No período de agosto de 2001 a agosto de 2003 foram estudados, prospectivamente, pacientes portadores de processos expansivos supra-selares com evidências neurorradiológicas de compressão atual ou pregressa da via óptica, no Serviço de Neuroftalmologia do Hospital das Clínicas da Faculdade de Medicina da Universidade de São Paulo.

Os pacientes foram submetidos a exame oftalmológico completo que incluiu medida da acuidade visual, exame refracional, avaliação da motilidade ocular, reações pupilares, biomicroscopia, tonometria de aplanação e fundoscopia. A seguir, foi realizado o exame de campo visual no perímetro de Goldmann e no perímetro Humphrey. O exame de fundo de olho sob midríase foi realizado após os exames de campo visual.

A perimetria computadorizada foi realizada com o perímetro Humphrey (Humphrey Systems, Dublin, CA, USA), utilizandose o programa "24-2 Full threshold test" com estímulo branco e mira III. O exame de campo visual manual foi realizado no perímetro de Goldmann (Haag-Streit AG, Bern, Switzerland), devidamente calibrado. Utilizaram-se também, em todos os pacientes, as miras V/4e, I/4e e I/2e. Em muitos casos utilizaram-se também as miras I/3e e I/1e. A apresentação cinética dos estímulos luminosos foi realizada para determinar as isópteras correspondentes. Os pacientes foram submetidos aos exames perimétricos manual e computadorizado no mesmo dia, após descanso apropriado, ou em outro dia com intervalo máximo de duas semanas entre os dois exames, desde que não tivesse sido realizado tratamento específico para a lesão neste período.
Dezenove pacientes foram selecionados para este estudo. Os pacientes foram selecionados com base no resultado do exame da perimetria computadorizada e para serem incluídos no estudo, deveriam apresentar, em pelo menos um olho, defeito perimétrico temporal completo (com a estratégia 24-2 full threshold) que respeitasse o meridiano vertical (Figura 1). Apenas os olhos com tais defeitos foram objetos de estudo, embora a perimetria tenha sido realizada nos dois olhos. Outros critérios de inclusão para os pacientes foram os seguintes:

1. Idade acima de 15 anos.

2. Ausência de anormalidades oculares, exceto a presença de erros de refração menores que seis dioptrias esféricas ou que três dioptrias cilíndricas.

3. Acuidade visual melhor que ou igual a $20 / 30$ no olho estudado.

4. Boa colaboração para o exame e disponibilidade para realizar a perimetria manual e computadorizada ou no mesmo dia, ou em outro dia com intervalo máximo de duas semanas entre eles.

5. Apresentar ou ter apresentado tumor supra-selar com compressão quiasmática, documentado ao exame de imagem.

Vinte e cinco olhos foram selecionados para análise. As perimetrias computadorizadas foram analisadas quanto ao valor do "mean deviation" (fornecido automaticamente pelo aparelho) e quanto às características gráficas observadas no he-

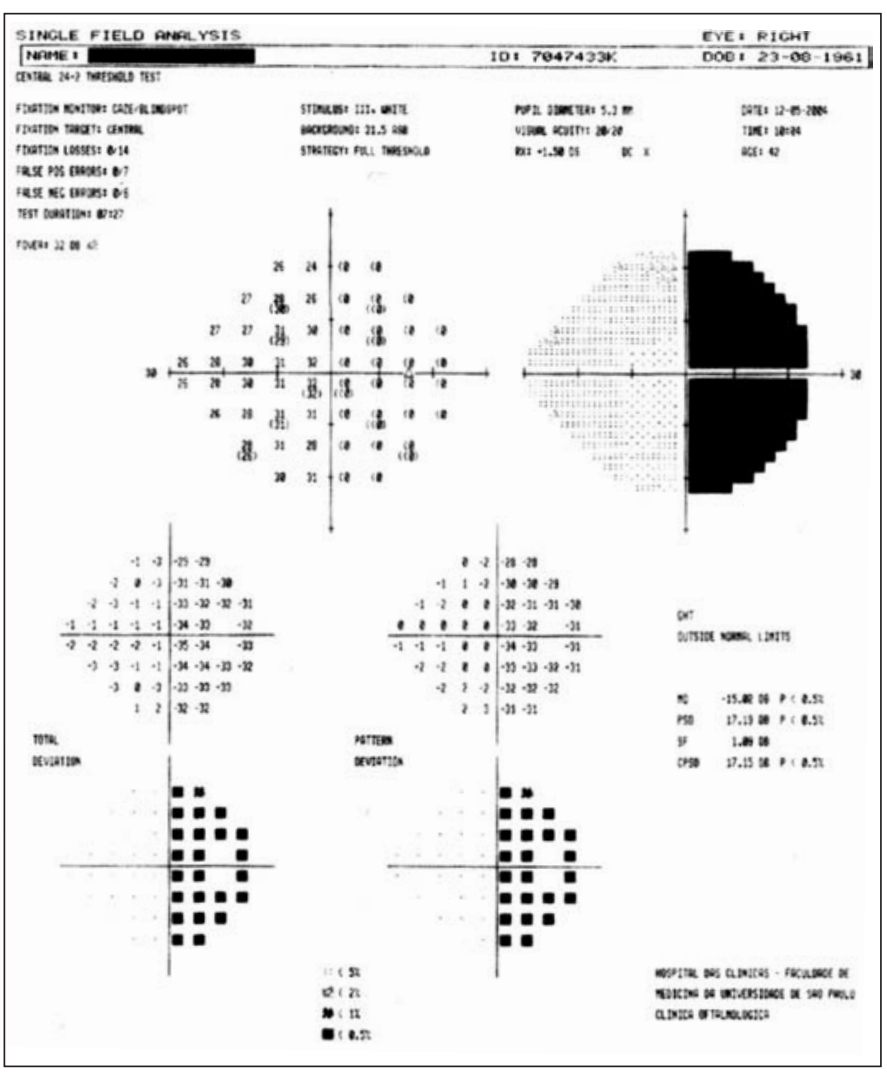

Figura 1 - Hemianopsia temporal completa à perimetria computadorizada Humphrey 24-2 Full threshold test, utilizada como critério de seleção para os olhos a serem estudados 
micampo temporal, tanto nos gráficos de probabilidades "total deviation" e "pattern deviation" como também no gráfico em tons de cinza. A seguir, foi avaliada a perimetria de Goldmann, com a finalidade de se verificar a presença ou não de visão remanescente do setor temporal do campo visual.

Os pacientes foram divididos em dois grupos: a) grupo 1, relativo a pacientes portadores de hemianopsia temporal completa ao Goldmann; b) grupo 2, relativo a pacientes portadores de visão remanescente no campo visual temporal, presentes ao Goldmann. Foi calculada a média do "mean deviation" de cada um dos dois grupos e os resultados comparados.

\section{RESULTADOS}

Oito pacientes eram do sexo feminino e 11 do masculino com

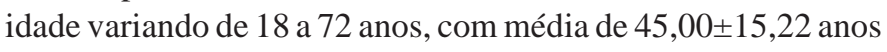
(média \pm desvio padrão). Quanto ao tipo de tumor, 14 eram portadores de adenoma hipofisário e 5 de craniofaringioma. No que diz respeito à situação do tumor, 4 pacientes apresentavam compressão ativa e 15 pacientes apresentavam defeito seqüelar. A tabela 1 demonstra os dados referentes ao sexo, à idade, ao olho estudado, ao tipo e à situação do tumor. A tabela 2 mostra os achados na perimetria computadorizada e manual.

$\mathrm{Na}$ análise das perimetrias computadorizadas verificamos que estas deixaram de evidenciar o remanescente temporal observado ao Goldmann em 17 (68\%) dos 25 olhos estudados (Figura 2). Outros 8 olhos apresentaram hemianopsia completa ao perímetro Humphrey e também ao perímetro de Goldmann (Grupo 1). Neste grupo o "mean deviation" variou entre-14,11 e

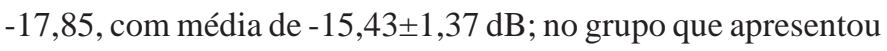
remanescente temporal no campo visual ao Goldmann (Grupo 2, composto por 17 olhos), o "mean deviation" variou de $-13,88$ a $-20,12$ com média de $-15,93 \pm 2,11 \mathrm{~dB}$. O estudo estatístico não revelou diferença significativa entre as médias do "mean deviation" apresentadas pelos dois grupos $(\mathrm{p}=0,54)$.

\section{DISCUSSÃO}

O exame do campo visual assume um papel relevante para o diagnóstico e acompanhamento dos pacientes com distúrbios neuroftalmológicos, em especial nos casos de tumores supra-selares.

Por muitos anos a perimetria de Goldmann foi considerada o método perimétrico ideal para ser aplicado nos pacientes com lesões neuro-oftalmológicas. O grande avanço tecnológico ocorrido nas últimas décadas transformaram a perimetria computadorizada no procedimento mais utilizado na avaliação dos campos visuais na atualidade, especialmente para a detecção de defeitos campimétricos decorrentes de glaucoma ${ }^{(1)}$. Entre as vantagens deste tipo de perimetria estão a sua reprodutibilidade e a possibilidade de se definir, através de índices numéricos, a sensibilidade de cada ponto, bem como de se realizarem comparações mais precisas ao longo do tempo.

Apesar das vantagens da perimetria computadorizada acima citadas, o papel da mesma nos casos de afecções neuroftalmológicas ainda é assunto de discussão ${ }^{(4)}$. Inúmeras são as condições neuroftalmológicas nas quais a perimetria computadorizada falha na avaliação dos campos visuais. Em um estudo

\begin{tabular}{|c|c|c|c|c|c|}
\hline Paciente & Sexo & Idade & Olho estudado & Tipo de tumor & Situação do tumor \\
\hline 1 & M & 43 & OE & Craniofaringeoma & Defeito seqüelar \\
\hline 2 & $\mathrm{~F}$ & 41 & OD & Craniofaringeoma & Defeito seqüelar \\
\hline 3 & M & 55 & $\mathrm{AO}$ & Adenoma & Defeito seqüelar \\
\hline 4 & M & 48 & $\mathrm{AO}$ & Adenoma & Defeito seqüelar \\
\hline 5 & M & 50 & $\mathrm{AO}$ & Adenoma & Defeito seqüelar \\
\hline 6 & $\mathrm{~F}$ & 61 & OD & Adenoma & Defeito seqüelar \\
\hline 7 & $\mathrm{~F}$ & 38 & OE & Adenoma & Defeito seqüelar \\
\hline 8 & $M$ & 72 & OE & Adenoma & Defeito seqüelar \\
\hline 9 & M & 47 & OD & Adenoma & Defeito seqüelar \\
\hline 10 & $\mathrm{~F}$ & 34 & OD & Adenoma & Defeito seqüelar \\
\hline 11 & M & 21 & $\mathrm{AO}$ & Adenoma & Defeito seqüelar \\
\hline 12 & M & 60 & OD & Adenoma & Defeito seqüelar \\
\hline 13 & M & 18 & OD & Craniofaringeoma & Defeito seqüelar \\
\hline 14 & $\mathrm{~F}$ & 23 & OD & Adenoma & Defeito seqüelar \\
\hline 15 & $M$ & 55 & OE & Adenoma & Defeito seqüelar \\
\hline 16 & $\mathrm{~F}$ & 35 & OE & Craniofaringeoma & Compressão ativa \\
\hline 17 & $\mathrm{~F}$ & 34 & $\mathrm{AO}$ & Adenoma & Compressão ativa \\
\hline 18 & M & 66 & OD & Craniofaringeoma & Compressão ativa \\
\hline 19 & $\mathrm{~F}$ & 54 & $\mathrm{AO}$ & Adenoma & Compressão ativa \\
\hline
\end{tabular}




\begin{tabular}{|c|c|c|c|}
\hline Olho & Paciente & MD & Campo manual \\
\hline 1 & 1 & $-15,52$ & Hemianopsia completa \\
\hline 2 & 2 & $-14,11$ & Hemianopsia completa \\
\hline 3 & 3 & $-14,04$ & Remanesc. temp. com V4e e TI com I4e \\
\hline 4 & 3 & $-18,23$ & Remanesc. temp. com V4e e TI com I4e \\
\hline 5 & 4 & $-15,97$ & Esc. hemianópico temp. e Remanesc. temp. com V4e e TI com I4e \\
\hline 6 & 4 & $-18,06$ & Remanesc. $\mathrm{TI}$ com V4e \\
\hline 7 & 5 & $-15,42$ & Hemianopsia completa \\
\hline 8 & 5 & $-14,15$ & Hemianopsia completa \\
\hline 9 & 6 & $-13,97$ & Remanesc. $\mathrm{Tl}$ com $\mathrm{V} 4 \mathrm{e}$ \\
\hline 10 & 7 & $-14,11$ & Remanesc. TI com mira V4e \\
\hline 11 & 8 & $-14,96$ & Remanesc. temp. com V4e e $14 \mathrm{e}$ \\
\hline 12 & 9 & $-14,00$ & Remanesc. $\mathrm{TI}$ com V/4e e I/4e \\
\hline 13 & 10 & $-15,04$ & Remanesc. temp. com $\mathrm{V} / 4 \mathrm{e}$ e $\mathrm{I} / 4 \mathrm{e}$ e Esc. temp. \\
\hline 14 & 11 & $-15,38$ & Remanesc. temp. V/4e \\
\hline 15 & 11 & $-17,85$ & Remanesc. $\mathrm{TI}$ com V/4e \\
\hline 16 & 12 & $-15,23$ & Hemianopsia completa \\
\hline 17 & 13 & $-20,12$ & Remanesc. $\mathrm{TI}$ com $\mathrm{V} / 4 \mathrm{e}$ e $\mathrm{I} / 4 \mathrm{e}$ \\
\hline 18 & 14 & $-16,99$ & Hemianopsia completa \\
\hline 19 & 15 & $-20,10$ & Remanesc. temp. com V4e \\
\hline 20 & 16 & $-17,85$ & Hemianopsia completa \\
\hline 21 & 17 & $-14,94$ & Esc. hemianópico com V4e e 14 e e Remanesc. temp. com V4e e 14 e \\
\hline 22 & 17 & $-15,45$ & Esc. hemianópico com V4e; Remanesc. temp. com V4e e Remanesc. TI com mira 14e \\
\hline 23 & 18 & $-14,79$ & Esc. hemianópico com V4e e Remanesc. temp. com V4e e $14 \mathrm{e}$ \\
\hline 24 & 19 & $-13,88$ & Esc. hemianópico com V4e e Remanesc. temp. com V4e \\
\hline 25 & 19 & $-14,19$ & Hemianopsia completa \\
\hline
\end{tabular}

recente, demonstramos que a simples análise do gráfico em tons de cinza deixa de identificar defeitos quiasmáticos discretos em um número considerável de pacientes ${ }^{(5)}$. Da mesma forma a observação dos pontos significativos nos gráficos de probabilidades "pattern deviation" e "pattern deviation" parece não ser suficiente para identificar defeitos quiasmáticos iniciais ${ }^{(5)}$. Outras importantes fontes de imprecisão na perimetria computadorizada ocorre nos pacientes com diminuição acentuada da acuidade visual, nos quais a confiabilidade do exame fica muito prejudicada; essa imprecisão também ocorre nos pacientes com lentidão nas respostas, ocasionada por dificuldades motoras ou cognitivas; neles, o exame de perimetria computadorizada fica freqüentemente muito prejudicada.

No presente estudo procuramos avaliar pacientes portadores de tumores supra-selares com compressão quiasmática ativa ou portadores de defeito seqüelar levando a defeitos severos de campo visual (hemianopsia temporal completa ao Humphrey), porém com acuidade visual preservada para tornar o exame computadorizado confiável. Pudemos observar que em $68 \%$ dos casos (17 dos 25 olhos estudados), o perímetro computadorizado realizado com a mira III e estratégia "24-2 full threshold" não identificou a presença de remanescente temporal periférico residual pelo fato de avaliar apenas a área central (Tabela 2, Figura 2). A possibilidade de avaliação simultânea da região central e periférica do campo visual oferece à perimetria de Goldmann uma vantagem extraordinária, seja na documentação mais exata da função visual, seja no auxílio no que se refere à realização do diagnóstico pela melhor compreensão do padrão do defeito de campo ${ }^{(3,6)}$. Este estudo mostra que, toda vez que o paciente apresentar hemianopsia completa ao perímetro computadorizado, a avaliação deve ser complementada com a realização da perimetria manual. Outra possibilidade seria a de se repetir a perimetria computadorizada utilizando uma mira maior (mira V) que poderia auxiliar na identificação de áreas remanescentes no campo de visão temporal. Esta possibilidade, ainda não comprovada cientificamente, necessita ser mais bem avaliada em estudos prospectivos específicos.

No estudo atual, procuramos comparar o "mean deviation" fornecido pelo aparelho nos pacientes com hemianopsia completa ao Goldmann com aqueles com remanescente temporal no Goldmann, no sentido de verificar se o valor do "mean deviation" poderia servir para separar estes dois grupos de pacientes. Observamos, no entanto, que as médias do mean deviation nos dois grupos foi muito semelhante, sendo -15,43 $\pm 1,37$, e -15,93 $\pm 2,11$ nos grupo 1 e grupo 2 , respectivamente. $\mathrm{O}$ estudo estatístico não demonstrou diferença significativa entre os dois grupos de pacientes estudados, indicando que a análise deste parâmetro não nos auxilia na identificação dos possíveis pacientes portadores de remanescente periférico de campo visual. 


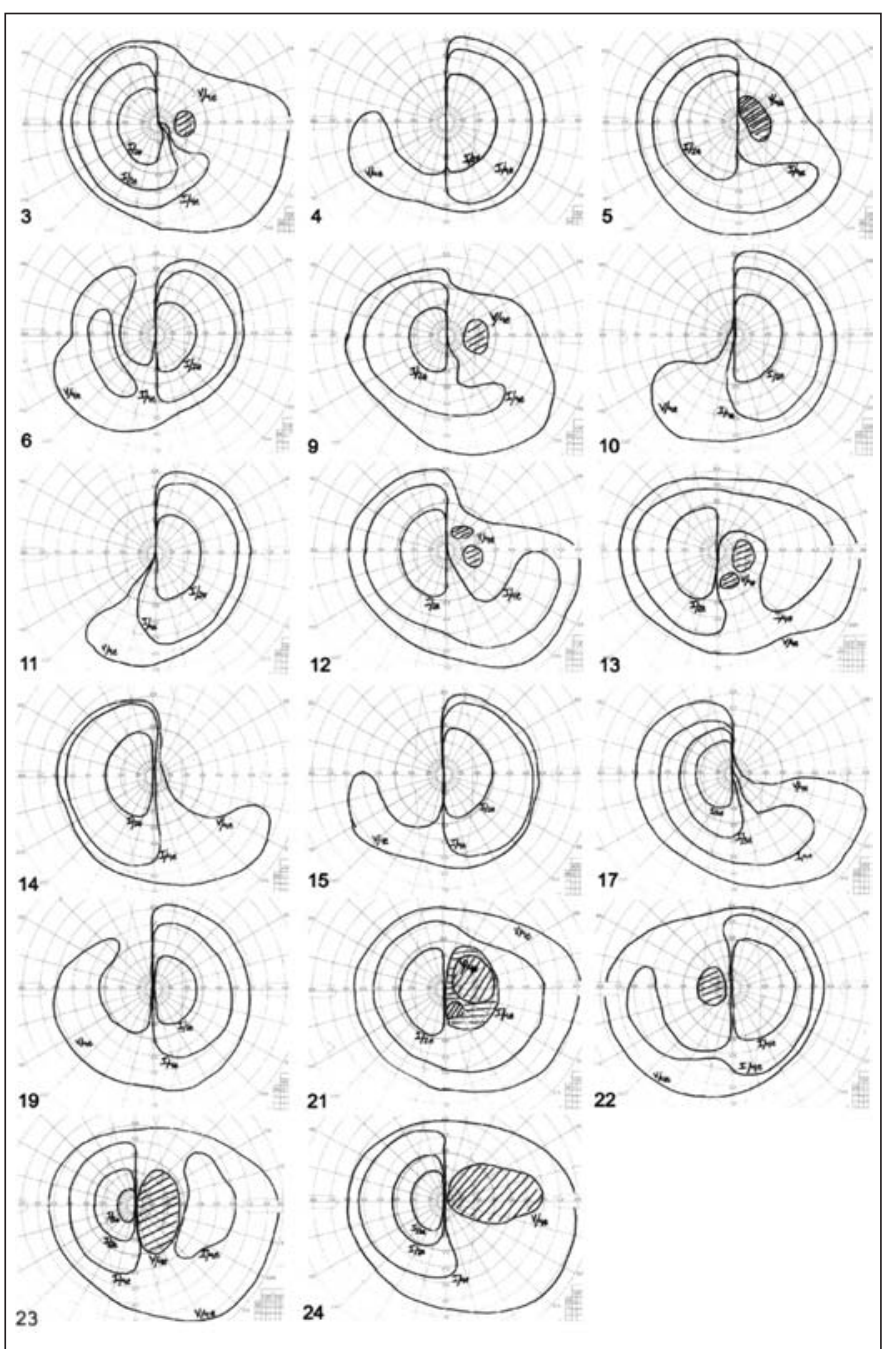

Figura 2 - Resultados da perimetria de Goldmann nos 17 olhos que apresentaram áreas remanescentes no campo visual temporal. Os números indicam os olhos enumerados na tabela 2

\section{CONCLUSÃO}

O estudo atual demonstrou que a perimetria computadorizada não consegue identificar os pacientes com remanescentes periféricos do campo visual, e o "mean deviation" fornecido pelo aparelho não nos auxilia na identificação de tais pacientes. Sendo assim, a perimetria computadorizada não substitui a perimetria manual nestes casos. Na indisponibilidade de perímetros manuais, salientamos a necessidade da realização de pelo menos um teste de confrontação para tentar identificar a presença de possíveis remanescentes periféricos no campo visual destes pacientes.

\section{ABSTRACT}

Purpose: To study, on Goldmann perimetry, a group of patients with complete temporal hemianopia on Humphrey perimetry (24-2 full threshold test) and to evaluate the percentage of eyes in which computerized perimetry failed to identify a temporal visual field remnants. Methods: Nineteen patients with visual field defect by chiasmal compression were prospectively studied with manual and automated perimetry. Twenty-five eyes with complete temporal hemianopia on Humphrey Field Analyzer, 24-2 threshold test were selected and studied with Goldmann perimeter, in order to evaluate the presence of a temporal visual field remnants. According to the result of Goldmann perimetry the eyes were divided into two groups: group 1, with complete temporal hemianopia; group 2, with residual temporal visual field remnants in the periphery. The mean number of mean deviation in the 2 groups was calculated and compared using Student's t test. Results: Automated perimetry failed to identify a temporal residual visual field in 17 of 25 eyes studied (68\%). Mean values of the mean deviation in groups 1 and 2 were respectively -15.43 and -15.93. Statistical analysis did not indicate a significant difference between them. Conclusions: Computerized automated Humphrey perimetry using 24-2 threshold test fails to identify temporal visual field remnants in a great percentage of patients with severe chiasmal compression. Analysis of the mean deviation is not helpful in identifying such cases. Patients evaluated on automated perimetry and presenting with a complete temporal hemianopia should be checked for temporal visual field remnants.

Keywords: Perimetry/methods; Visual fields; Optic chiasm/ pathology; Vision disorders/diagnosis; Pituitary Neoplasms/ diagnosis; Diagnostic techniques, ophthalmological

\section{REFERÊNCIAS}

1. Katz J, Tielsch JM, Guigley HA, Sommer A. Automated perimetry detects visual field loss before manual Goldmann perimetry. Ophthalmology. 1995; 102(1):21-6.

2. Beck RW, Bergstrom TJ, Lichter PR. A clinical comparison of visual field testing with a new automated perimeter, the Humphrey field analyzer, and Goldmann perimeter. Ophthalmology. 1985;92(1):77-82.

3. Wong AM, Sharpe JA. A comparison of tangent screen, Goldmann and Humphrey perimetry in detection and localization of occipital lesions. Ophthalmology. 2000;107(3):527-44. Comment in: Ophthalmology. 2001;108(2):214-2.

4. Grochowicki M, Vighetto A, Berquet S, Khalfallah Y, Sassolas G. Pituitary adenomas: automatic static perimetry and Goldmann perimetry. A comparative study of 345 visual field charts. Br J Ophthalmol. 1991;75(4):219-21.

5. Monteiro MLR, Pereira A. Perimetria computadorizada na detecção de defeitos campimétricos discretos (ao perímetro de Goldmann) causados por tumores hipofisários. Arq Bras Oftalmol. 2003;66(6):803-9.

6. Pollock SC. Visual fields in optic neuritis. Arch Ophthalmol. 1993;111(1):1315-7. 